\title{
Research Paper: The Asthenozoospermia as a Major Sperm Abnormality in Males Assessed for Semen Analysis in Institut Pasteur of Cote d'Ivoire
}

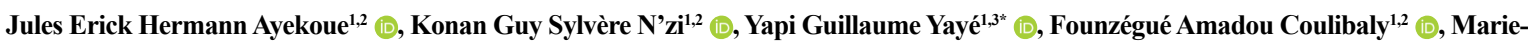

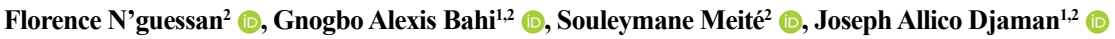

1. Pharmacodynamic and Biochemistry Laboratory, UFR Biosciences, University of Félix Houphouët-Boigny, Abidjan, Ivory Coast.

2. Department of Medical Biochemistry, Institute of Ivory Coast, Abidjan, Ivory Coast.

3. Department of Biochemistry and Microbiology, UFR Agroforestry, University of Jean Lorougnon Guédé, Daloa, Ivory Coast.

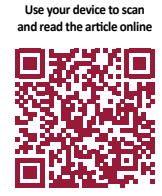

Citation Ayekoue JEH, N'zi KGS, Yayé YG, Coulibaly FA, N'guessan MF, Bahi GA, et al. The Asthenozoospermia as a Major Sperm Abnormality in Males Assessed for Semen Analysis in Institut Pasteur of Cote d'Ivoire. JAMSAT. 2018; 4(1):7-12. https://doi.org/10.32598/jamsat.4.1.7

https://doi.org/10.32598/jamsat.4.1.7

Keywords:

Asthenozoospermia, Stress, Infertility, Spermogram, Spermocytogram

\section{ABSTRACT}

Objectives: The male infertility is on the increase worldwide; therefore, it is attracting more attention in several studies in order to improve its treatment management. The current study aimed at contributing to set up epidemiological and biological data and draw a general profile of male infertility in Côte d'Ivoire.

Materials \& Methods: The current prospective and descriptive study was conducted on 401 males`sperm obtained through masturbation after three days of abstinence. The analysis of the information in the cards accompanying the samples and the spermogram and spermocytogram analyses were used to collect the related data.

Results: The average age of the males in the study was 39.97 years and the average duration of infertility was 6.66 years; $35.16 \%$ of the subjects were in the private sector and mostly composed of traders. Primary infertility was the leading cause of consultation in $55.61 \%$ of the cases. A significant relationship $(\mathrm{P}=0.03)$ between stress and azoospermia was highlighted, showing that males with stress were twice as likely to develop azoospermia (Odds Ratio=2.24). Results of the current study showed that in the vast majority, semen had several abnormalities with a high rate of associated abnormalities, $37.41 \%$. However, among the isolated abnormalities, asthenozoospermia was the most prominent abnormality with a rate of $20.70 \%$.

Conclusion: This study shows that the sperms evaluated have many abnormalities, the most important of which is asthenozoospermia.

\section{* Corresponding Author:}

Yapi Guillaume Yayé, PhD.

Address: Pharmacodynamic and Biochemistry Laboratory, UFR Biosciences, University of Félix Houphouët-Boigny, Abidjan, Ivory Coast.

Tel: +2 (25) 08114855

E-mail: yayeyapi@yahoo.fr 


\section{Highlights}

- The vast majority of sperms show some abnormalities in infertile men.

- Asthenospermia (poor mobility of sperm) is the most important observed abnormality.

- Stress seems to be a risk factor associated mainly with azoospermia (no sperm in).

\section{Plain Language Summary}

Male infertility is one of the pathologies found in couples with difficulty in having child naturally. Nowadays, infertility equally affects women and men. In Ivory Coast, this pathology is frequently observed. It is therefore important to look into this problem (male infertility) to improve the management of these patients. In this regard, the patients referred to the Institute Pasteur in Ivory Coast for the purpose of their fertility assessment were enrolled in this study. Most patients had not yet had children and therefore suffered from primary infertility. Their sperms were collected according to WHO laboratory manual (2010, 5th edition) for the examination. Most of the analyzed sperms had several abnormalities, the most important of which is asthenospermia (poor mobility of sperm). Male infertility affects men with different occupations with a predominance of small businesses and civil servants. Moreover, the study also revealed that one of the major risk factors for infertility is stress and this one would be mainly related to azoospermia (no sperm in semen).

\section{Introduction}

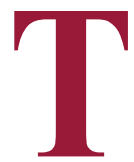

he desire to have a kid is one of the major projects of everybody, whether in a relationship or not. However, more than 80 million people or $10 \%$ to $15 \%$ of the world adult population are affected by infertility [1]. Indeed, the infertility is defined as the inability of the sexually active couple, not taking contraception, to have a pregnancy within one year [2].

It is not only a medical problem but also a social crisis by influencing interpersonal, conjugal, and social relationships and creating psychological damage [3]. Infertility is a global issue with a prevalence of $5 \%$ to $30 \%$ [4]. Although the estimates are not precise and the figures vary from one region to another, in Africa, $20 \%$ to $30 \%$ of couples have infertility during their period of genital activity with a high prevalence rate around $35 \%[5,6]$. Regarding male infertility, this is a recent finding, as in the past, only women were accused of infertility [7]. It represents about 30\% to $50 \%$ of cases of infertility of the couple with $10 \%$ to $20 \%$ of unexplained cases. This is the case of Côte d'Ivoire, a region with a high prevalence but no real data source [8].

According to the high rate, scientific research is interested in this type of infertility. It is therefore necessary to pay special attention to identify and understand the different spermatic anomalies that can be found in humans. It allows good management of the suffering of the couples that want to have children. In addition to the lack of data constituting a considerable obstacle to improving the therapeutic management of spermatic anomalies, the current study aimed at establishing a general state of male infertility in Côte d'Ivoire based on epidemiological and biological data.

\section{Material and Methods}

\section{Type and Scope of study}

The current prospective and descriptive study was conducted in the laboratory of Biological Reproduction Unit (BRU) of Institut Pasteur of Côte d'Ivoire (IPCI).

\section{Biological material}

The study included sperm samples taken from males referring for a fertility test from April 2017 to September 2017 (six months).The sperm was collected on site (in the laboratory) by masturbation after abstinence for 3 days, according to the recommendations of the WHO 2010. The epidemiological data included in the information sheets accompanying the semen ejaculates for routine examinations were completed with informed consent from the persons concerned.

The data consisted of age, occupation, type (primary and secondary) and duration of infertility (time to conceive a child), and risk factors such as STI, alcohol, tobacco, and stress. In addition, the biological parameters including sper- 
mogram and spermocytogram were studied in order to assess the quality of the sperm.

\section{Biological study}

First, macroscopic data including volume, color, viscosity and $\mathrm{pH}$ were determined. Indeed, after the sperm collection, the semen was placed in an incubator at $37^{\circ} \mathrm{C}$ for one hour to be liquefied. Then using a graduated pipette, the volume was measured. As for the viscosity, it was evaluated using a dropper pipette. Color appreciation was done with the naked eyes, and a $\mathrm{pH}$ indicator paper (MACHEREY-NAGEL) was used to determine the $\mathrm{pH}$ of the spermatic liquid.

Secondly, the microscopic data including the number of spermatozoa, vitality, number of round cells, mobility and sperm cytology were assessed. Thus, after liquefaction (1 hour after sampling), the determination of the vitality, sperm count, and motility of the spermatozoa was conducted by an automated Sperm Quality Analyzer (SQA-Vision) with an operating principle based on the combination of optics and information technology system. In addition, the round cells were counted after mounting a drop of sperm between slide and coverslip under a microscope. Cytology was carried out with the RAL 555 kit. Thus, the percentage of typical shape and different shape anomalies (head, intermediate piece, main piece, and cytoplasmic remainder) were evaluated according to the Kruger and Menkveld criteria admitted by WHO, 2010 [2], which allow the determination of the Teratozoospermia Index (TZI). In addition, the analysis of the spermogram and the spermocytogram helped to identify different spermatic profiles according to the WHO 2010 standards [2].

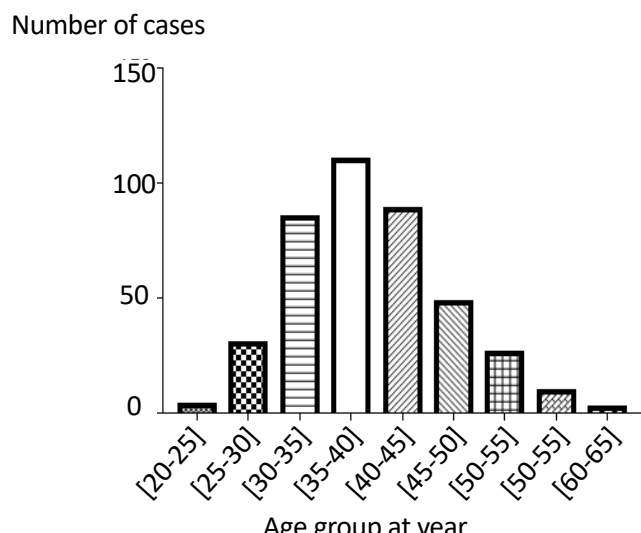

Figure 1. Distribution of patients by age group

\section{Data analysis}

The XLSTAT 2014 software was used to analyze the data and the level of significance was $\mathrm{P}<0.05$. The value of the etiological role of the risk factors was determined based on the values of Relative Risk (RR) and Odds Ratio (OR). Thus, for values of $\mathrm{RR}$ and $\mathrm{OR}=1$ there was no relationship between the entities, but there was an association between entities if the values of $R R$ and $O R>1$.

\section{Results}

A total of 401 fact sheets corresponding to the number of semen samples received were analyzed. The average age of the subjects was $39.97 \pm 7.22$ years within the range of 2264 years. The grouping by age revealed that the age group $35-40$ years predominated with 110 cases $(27.43 \%$ ) (Figure 1). These patients mostly worked in the private sector (141 cases, 35.16\%) and mainly owned small businesses. This group was followed by that of the civil servants, 115 cases $(28.68 \%)$. In addition, analysis of the collected information also highlighted the predominance of primary infertility in 223 cases $(55.61 \%)$ compared to 178 cases $(44.39 \%)$ for secondary infertility. The average duration of infertility was $6.66 \pm 5.52$ years ranging 1 to 40 ; and the predominant subjects $(54.11 \%$; 217.401) in this case were the ones going through their first five years of infertility (Figure 2).

Biologically, of the 401 received sperm samples, 381 (95.01\%) had spermogram and spermocytogram values disrupted versus only $20(4.99 \%)$ normosperms. In the vast majority, semen had several abnormalities with a high rate of associated abnormalities, $37.41 \%$. However, among the isolated abnormalities, asthenozoospermia was the most prominent one with a rate of $20.70 \%$ (Figure 3 ).

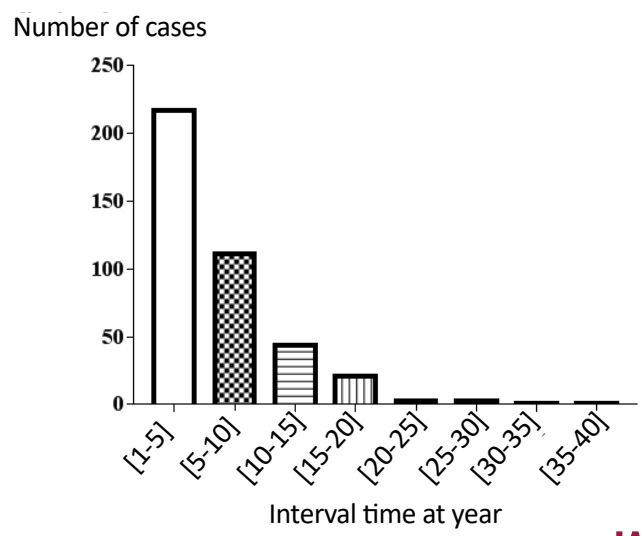

JAMSAT

Figure 2. Distribution of patients according to duration of infertility 

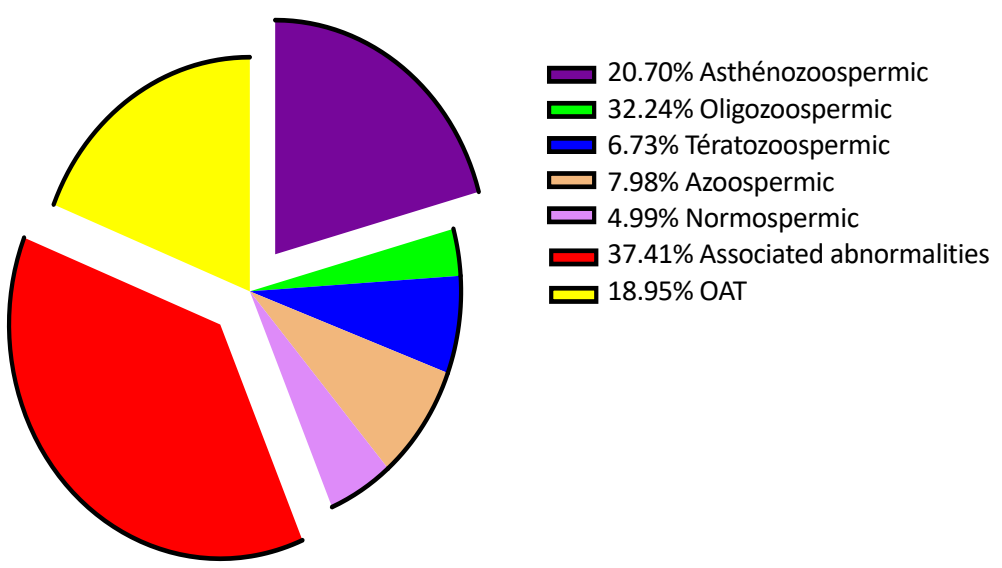

OAT: Oligo Astheno Teratozoospermia

Associated abnormalities: associating two by two between oligozoospermia, asthenozoospermia and teratozoospermia

Figure 3. Distribution of sperm abnormalities

JAMSAT

The current study also revealed associations between risk factors and sperm abnormalities highlighted by RR and OR values greater than 1 (Table 1).

\section{Discussion}

The average age of the study subjects was 39.97 years and that the most predominant age group was $35-40$ years. The obtained results were similar to those of Niang et al. [9] in Senegal, Moussa et al. [10] in Niger and Ouegnin et al. in Côte d'Ivoire [11]. This could be due to the marriage age, which is high in recent years in Africa, which results from socioeconomic status $[12,13]$.

The lowering of sperm quality is observed with advancing age and the type of occupation. Concerning the age, Dunson et al. found that the percentage of males 35-40 years old that cannot having a child before 12 months increases by about $23 \%$ per year [14]. As for the occupation, the samples from owners of small businesses and administrators were the most affected ones, respectively. These results could be explained by the sedentary or semi-sedentary activities responsible for the disruption in the blood circulation related to prolonged sitting as well as testicular warming, which affect spermatogenesis and sperm quality [10].

The results of the duration of infertility, which ranges from 1 to 40 years and the mean duration of 6.66 years were consistent with those of Niang et al. in Senegal and Ikechebelu et al. in Nigeria [9, 15]. As in the case of age, these results could be explained by the more and more degrading quality of sperm observed $[6,16]$.

Regarding the type of infertility, the study showed that primary infertility was the first cause of the demand for the fertility test; showing the increasing interest that the

Table 1. Relationship between risk factors and spermatic abnormalities revealed

\begin{tabular}{cccccc}
\hline Risk Factor & Abnormality & Relative Risk & Odds Ratio & Chi-Square Test & $\mathbf{P}$ \\
\hline \multirow{2}{*}{ STI } & Oligozoospermia & 1.13 & 1.13 & 0.05 & 0.83 \\
& Azoospermia & 1.19 & 1.21 & 0.27 & 0.60 \\
\multirow{2}{*}{ Alcohol } & Asthenozoospermia & 1.16 & 1.20 & 0.57 & 0.45 \\
& Associated abnormalities & 1.08 & 1.13 & 0.34 & 0.56 \\
Tobacco & OAT & 1.59 & 1.84 & 1.26 & 0.26 \\
& Azoospermia & 2.08 & 2.24 & 4.83 & 0.03 \\
Stress & OAT & 1.39 & 1.52 & 1.92 & 0.17 \\
\hline
\end{tabular}

STI: Sexually Transmitted Infection; OAT: Oligo Astheno Teratozoospermia 
populations have in their reproductive health. Moreover, since the males who already have a kid (secondary infertility); find it difficult to accept the idea of being responsible for their couple's infertility. They prefer to impute it to their spouse $[1,12]$.

From the point of the biological assessment, the study showed that in $95.01 \%$ of the received semen samples, the spermograms and spermocytograms were disrupted against only $4.99 \%$ of normospermia. This strong disruption of sperm justifies the growing number of male consulting for infertility problems. Indeed, similar longitudinal studies in Senegal [9], Nigeria [15], and Côte d'Ivoire [11] show the increasing trend of consultations for infertility. Thus, among the observed abnormalities, the predominance of associated abnormalities (37.41\%) was revealed, which could be explained by the multifactorial etiology of male infertility. However, the abnormalities taken separately showed that asthenozoospermia was the most observed one (20.70\%). Indeed, the motility abnormality is one of the most observed causes of male infertility during the fertility test [17]. It is generally due to the deficit of the operation of the flagellum due to the complex mechanism of the acquisition of the motility of the spermatozoa in the epididymis [18].

This abnormality may also be associated with a lack of ATP production required for flagellar movement [19]. This production of ATP is by the mechanism of oxidative phosphorylation within the mitochondria. It is provided by subunits of enzymatic complexes under the control of mitochondrial DNA. According to the respiratory chain, mitochondria are the site of production of Reactive Oxygen Species (ROS), which is a factor causing deficits in the functioning of these complexes [20]. In addition, when risk factors are associated with sperm abnormalities, a relationship between these two entities appears as RR and OR $>1$. Indeed the influence of risk factors such as STIs, alcohol, tobacco and stress on male infertility is described by several studies showing the plurality of causes of male infertility $[11,21,22]$. These risk factors that are the root of the environment-related infertility are linked to lifestyle or socio-economic situation. They result from functional deficiency of Leydig and Sertoli cells, resulting in androgenic insufficiencies and disruption of spermatogenesis.

The current study revealed that males with stress were two times more likely to develop azoospermia compared to the ones in normal condition. Indeed, the socio-economic problems underlying the stress could modify the secretion of GnRH in the hypothalamus, thus disrupting the hormonal functioning involved in the production and integrity of spermatozoa [23].

\section{Conclusion}

Male infertility is a recurring problem that requires special attention. In the developing countries, it is still badly perceived due to the confusion made between manhood and the ability to conceive. And yet almost all spermograms and spermocytograms are strongly disrupted with a predominance of sperm motility abnormalities (asthenozoospermia). It is therefore important to educate the population to avoid late awareness, which is very often the reason for therapeutic failure. Moreover, today the genetic exploration of the causes of sperm abnormalities through the determination of biological markers is essential to improve the management of male infertility.

\section{Ethical Considerations}

\section{Compliance with ethical guidelines}

The study was approved by the National Council of Ethics and Research of the country (reference number: 036/ MSLS/CNER/TB) and was conducted in accordance to the legal and regulatory provisions of Helsinki Declaration.

\section{Funding}

The present paper was extracted from the thesis.

\section{Authors contributions}

Conceptualization: Jules Erick Hermann Ayekoue and Joseph Allico Djaman; Investigation: Jules Erick Hermann Ayekoue and Konan Guy Sylvère N'zi1; Methodology: Jules Erick Hermann Ayekoue, Konan Guy Sylvère N'zi1, Yapi Guillaume Yayé, Joseph Allico Djaman; Formal analysis: Jules Erick Hermann Ayekoue, Konan Guy Sylvère N'zi1, Marie-Florence N'guessan; Writing, original draft: Jules Erick Hermann Ayekoue; Writing: Konan Guy Sylvère N'zi1 and Yapi Guillaume Yayé; Validation: Yapi Guillaume Yayé, Founzégué Amadou Coulibaly, Marie-Florence N'guessan, Gnogbo Alexis Bahi, Souleymane Meité, Joseph Allico Djaman; Supervisor: Founzégué Amadou Coulibaly, Souleymane Meité, Joseph Allico Djaman; Formal analysis: Founzégué Amadou Coulibaly; and Solfware: Jules Erick Hermann Ayekoue and Gnogbo Alexis Bahi.

\section{Conflict of interest}

The authors declared no conflict of interest.

\section{Acknowledgements}

The authors would like to express their deepest gratitude to the entire staff for the Clinical and Fundamental Bio- 
chemistry Department of IPCI and the reception and sampling unit (URAP) of IPCI.

\section{References}

[1] Vatin MM. [Study of genes involved in fertility human from a mouse model minterspecific recombinant congenic (IRCS) (French)] [MSc. thesis]. Paris: University Paris Descartes; 2008.

[2] World Health Organization. Laboratory manual for the examination and processing of human semen. Geneva: World Health Organization; 2010

[3] Karimi FZ, Taghipour A, Roudsari RL, Kimiaei SA, Mazlom SR, Amirian M. Cognitive emotional consequences of male infertility in their female partners: A qualitative content analysis. Electronic Physician. 2015; 7(7):1449-57. [DOI:10.19082/149] [PMID] [PMCID]

[4] Jafarzadeh Kenarsari F, Ghahiri A, Habibi M, Zargham Boroujeni A. Exploration of infertile couples' support requirements: A qualitative study. International Journal of Fertility \& Sterility. 2015; 9(1):81-92. [Doi:10.22074/ijfs.2015.4212] [PMID] [PMCID]

[5] Nana PN, Wandji JC, Fomulu JN, Mbu RE, Leke RJI, Woubinwou MJ. [Psychosocial aspects at infertile patients at the main maternity Central Hospital of Yaounde, Cameroon (French)] Clinics in Mother and Child Health. 2011; 8(1):1-5. [DOI:10.4303/ cmch/C100601]

[6] Eze UA, Okonofua FE. High prevalence of male infertility in Africa: Are mycotoxins to blame. African Journal of Reproductive Health. 2015; 19(3):9-17. [PMID]

[7] Traore M, Toure A, Sissoko S. [Spermiological profile of infertile men in Mali (French)]. Andrologie. 2008; 18(4):253-7. [DOI:10.1007/BF03040718]

[8] Naqvi H, Jaiswar SP, Hussain SR, Ahmad MK, Mahdi F, Mahdi AA. Genomic analysis of missense and silent mutations in CatSper 2 gene in infertile male cases. International Journal of Biomedical Research. 2016; 7(8):588-93.

[9] Niang L, Ndoye M, Labou I, Jalloh M, Kane R, Diaw JJ, et al. [Epidemiological and clinical profile of male infertility at GrandYoff General Hospital, Senegal: About 492 cases (French)]. Basic and Clinical Andrology. 2009; 19(2):103-7. [DOI:10.1007/s12610009-0019-x]

[10] Moussa D, Soumana A, Amadou SM, Soli I, Tahirou I, Ali A. [Hormonal profile in humans in the event of infertility at the radio immunology laboratory of the Institute of Radioisotopes of Niamey (French)]. African Journal of Urology. 2016; 22(4):305-9. [DOI:10.1016/j.afju.2016.01.008]

[11] Ouegnin GA, Gnagne YM, Vodi CC, Faye kette H, N’Douba KA, Tchetche RG. [Role of germs of genital infection in male infertility (French)]. Revue Bio-Africa. 2008; 6:32-38.

[12] Sankare O. [Contribution to the study of etiological aspects of male infertility at the service of cytogenetics and reproductive biology (French)] [MSc. thesis]. Mali: University of Bamako; 2008.

[13] Antoine P, Marcoux R. Plurality of marital forms and models in Africa: A state of play: introduction. In: Antoine P, Marcoux
R, editors. [Le Marriage in Africa: Plurality of forms and patterns of marriage (French)]. Presses of Laval University; 2014 [DOI:10.2307/j.ctt1f117sb.4]

[14] Dunson DB, Baird DD, Colombo B. Increased infertility with age in men and women. Obstetrics \& Gynecology. 2004; 103(1):51-6. [DOI:10.1097/01.AOG.0000100153.24061.45] [PMID]

[15] Ikechebelu JI, Adinma JIB, Orie EF, Ikegwuonu SO. High prevalence of male infertility in southeastern Nigeria. Journal of Obstetrics and Gynecology. 2003; 23(6):657-9. [DOI:10.1080/0144 3610310001604475] [PMID]

[16] Carlsen E, Giwercman A, Keiding N, Skakkebæk NE. Evidence for decreasing quality of semen during past 50 years. British Medical Journal. 1992; 305(6854):609-13. [DOI:10.1136/ bmj.305.6854.609] [PMID]

[17] Liu SW, Li Y, Zou LL, Guan YT, Peng S, Zheng LX, et al. Chloride channels are involved in sperm motility and are downregulated in spermatozoa from patients with asthenozoospermia. Asian Journal of Andrology. 2017; 19(4):418-24. [DOI:10.4103/1008-682X.181816] [PMID] [PMCID]

[18] Brugnon F. (Sperm apoptosis and male fertility (French)] [MSc. thesis]. Paris: Blaise Pascal University; 2009.

[19] May Panloup P, Chrétien MF, Malthiery Y, Reynier P. [Mitochondrial DNA of spermatozoa (French)]. Gynecology Obstetrics \& Fertility. 2006; 34(9):847-54. [DOI:10.1016/j.gyobfe.2006.06.016] [PMID]

[20] Migdal C, Serres M. [Reactive oxygen species and oxidative stress (French)]. Medicine/Science. 2011; 27(4):405-12. [DOI:10.1051/medsci/2011274017] [PMID]

[21] Marinelli D, Gaspari L, Pedotti P, Taioli E. Mini-review of studies on the effect of smoking and drinking habits on semen parameters. International Journal of Hygiene and Environmental Health. 2004; 207(3):185-92. [DOI:10.1078/1438-4639-00283] [PMID]

[22] Brzakowski M, Lourdel E, Cabry R, Olieric MF, Claeys C, Devaux A, et al. [Epidemiology of the infertile couple (French)] Journal of Gynecology Obstetrics and Biology of Reproduction. 2009; 38(1):F3-F7. [DOI:10.1016/S0368-2315(09)70226-1]

[23] Ravel C, Chantot Bastaraud S, El Houate BH, Rouba M, Legendre D, Lorenço, et al. Y-chromosome AZFc structural architecture and relationship to male fertility. Fertility and Sterility. 2009; 92(6):1924-33. [DOI:10.1016/j.fertnstert.2008.08.135] [PMID] 